\title{
Vertically Coupled InP/InGaAsP Microring Lasers Using a Single Epitaxial Growth and Single-Side Lithography
}

\author{
Óscar García López, Dries Van Thourhout ${ }^{\circledR}$, Member, OSA, Steven Verstuyft, \\ Manuel López-Amo ` , Senior Member, IEEE, Member, OSA, Roel Baets ${ }^{\circledR}$, Fellow, IEEE, Fellow, OSA, \\ and Marko Galarza ${ }^{(1)}$
}

\begin{abstract}
The experimental demonstration of vertical microring lasers requiring only one single epitaxial growth and two single-side lithographic steps is presented, in what is the simplest fabrication scheme for such devices published to date. The fabricated lasers show series resistance of around $20 \Omega$ at forward bias, threshold currents at room temperature between 35 and $58 \mathrm{~mA}$, and single-mode emission with a side-lobe suppression ratio higher than $30 \mathrm{~dB}$. The measured optic output power level is of tens of microwatts. The approach allows the improvement of the optical features maintaining the simplicity of the manufacturing procedure.
\end{abstract}

Index Terms-Integrated optics, ring lasers, semiconductor waveguides, semiconductor lasers.

\section{INTRODUCTION}

$\mathbf{O}$ PERATING similar to Fabry-Pérot resonators, but without the need of quality edge mirrors, microring resonators provide a sharp wavelength selectivity and a large field enhancement in a compact arrangement. Over the last years, they have been a fundamental element in a wide variety of high-performance photonic integrated devices providing optical functionalities as diverse as filtering [1], lasing [2], sensing [3], modulation [4], routing [5], switching [6], frequency comb generation [7], logic gates [8] and memories [9].

The basic layout of a ring resonator comprises a circular waveguide and one or two lateral waveguides operating as

Óscar García López is with the Deptartment of Electrical Electronic and Communication Engineering, Universidad Pública de Navarra, 31006 Pamplona, Spain, and also with the Centro Tecnológico de Automoción y Mecatrónica, 31006 Pamplona, Spain (e-mail: oscar.garcia@ naitec.es).

Manuel López-Amo and Marko Galarza are with the Deptartment of Electrical, Electronic and Communication Engineering, Universidad Pública de Navarra, 31006 Pamplona, Spain (e-mail: mla@ unavarra.es; marko.galarza@ unavarra.es).

Dries Van Thourhout, Steven Verstuyft, and Roel Baets are with the Department of Information Technology, Photonics Research Group, Ghent University-IMEC, 9052 Ghent, Belgium (e-mail: dries.vanthourhout@ugent. be; steven.verstuyft@ugent.be; roel.baets@ugent.be). evanescent wave input and output couplers to the ring. Although being a simple structure, the high quality factors required by the microrings imply very demanding fabrication challenges regarding the propagation loss in the ring and the coupling coefficient between the resonator and the bus waveguides. In the lateral coupling approach, the gap has to be submicron, usually requiring electron beam lithography. Moreover, since the microring and bus waveguides lay in the same epitaxial plane, the realization of functionalities that require active-passive integration is very complex. On the other hand, the vertical coupling approach containing two vertically stacked waveguide core layers, one to define the bus waveguides and the other for the microring, does allow for the definition of active-passive structures. Since the coupling between the bus and the ring waveguides, mainly depends on the thickness of the intermediate layer, which is accurately determined by the epitaxial growth, it can be controlled more precisely than on laterally coupled microring resonators. Nevertheless, wafer bonding or regrowth is usually required in order to manufacture such devices, increasing the complexity of their fabrication [10]. Furthermore, since two lithography steps are required either on each side of the wafer or after each growth step, alignment becomes a critical issue [11]. Either way, in both cases microring resonators have a definitive impact on the fabrication process of the devices of which they are part. Because of these technological specificities, the most developed approaches for $\mathrm{InP}$ monolithic integration do not include microring resonators as one of their basic building blocks. Butt-joint integration [12] allows the fabrication of ring lasers with larger cavities, but requires an inefficient all active approach for the coupling junction in the case of microrings [13]. The asymmetric twin-guide (ATG) technology [14], [15] neither reaches the compactness of microrings, as they have to include the gain section and two vertical tapers in the resonator [16]. The same issues apply to membrane-based schemes [17] since they also make use of a vertical integration arrangement where active and passive layers couple strongly [18].

We proposed in [19] a versatile active-passive integration approach, structurally similar to the ATG platform, but grounded on two symmetric vertical layers instead. The concept is technologically compatible with the component library of the ATG platform, since it is also based on vertical transitions between active and passive waveguides, although being in this case 


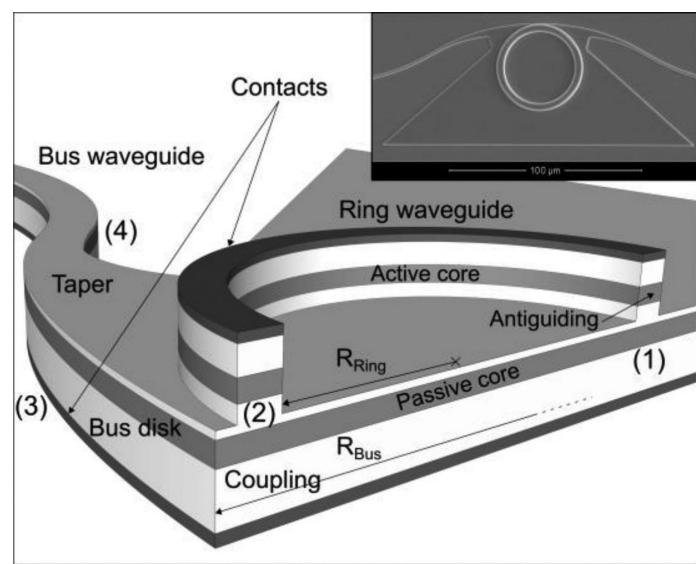

(a)

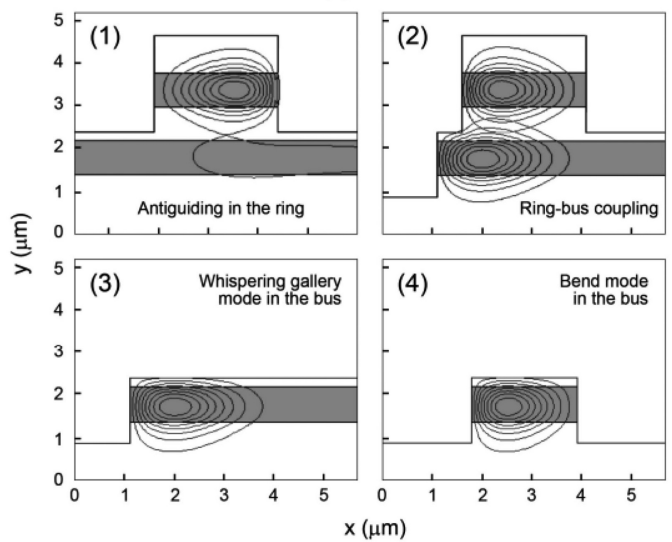

(b)

Fig. 1. (a) 3D schematic picture of the fabricated microring laser. In the inset, a photograph of the fabricated device before the metallization step. (b) Field distribution of the propagating modes in the ring, coupling region, bus disk, and bus waveguide.

compatible with the design of microring resonators as well. The theoretical proposal for the development of microring resonators using this structure was published in [20]. In the current work, the proof of concept of regrowth- and wafer-bonding-free vertically coupled microring lasers is presented, which increases the set of components offered by the earlier mentioned platforms.

\section{DeVice Structure And FAbrication}

Fig. 1(a) shows the 3D representation of the fabricated laser structure together with a top view photograph of the device prior to metallization. The key elements in the design of the laser are the two vertically coupled cores close to phase matching condition. The lower layer is passive and defines the bus waveguide, whereas the upper core is active and provides the guiding and the gain in the ring. Fig. 1(b) shows the field distribution of the propagation modes in each relevant section of the device. Light generated in the ring (1) excites the mode with the higher confinement in the active core, which is not the main even mode of the structure but the odd quasi-guided supermode. The bend loss of this strongly asymmetric mode shows an oscillatory pattern with minima below $0.1 \mathrm{~dB} / 90^{\circ}$ for radii larger than $100 \mu \mathrm{m}$, evolving under $1 \mathrm{~dB} / 90^{\circ}$ for smaller radii. The mode in
TABLE I

DESCRIPTION, COMPOSITION, THICKNESS AND DOPING LEVEL OF THE EPITAXIAL LAYER STACK

\begin{tabular}{ccccc}
\hline \hline Description & Composition & $\begin{array}{c}\text { Bandgap } \\
(\mu \mathrm{m})\end{array}$ & $\begin{array}{c}\text { Thickness } \\
(\mathrm{nm})\end{array}$ & Doping \\
\hline Contact layer & $\mathrm{In}_{0.53} \mathrm{Ga}_{0.47} \mathrm{As}$ & 1.65 & 160 & $\mathrm{p}++$ \\
p-contact & $\mathrm{InP}$ & 0.92 & 1000 & $\mathrm{p}++$ \\
p-contact & $\mathrm{InP}$ & 0.92 & 75 & $\mathrm{p}+$ \\
SCH & $\mathrm{In}_{0.83} \mathrm{Ga}_{0.17} \mathrm{As}_{0.37} \mathrm{P}_{0.63}$ & 1.13 & 190 & $\mathrm{nid}$ \\
Barrier $(\times 5)$ & $\mathrm{In}_{0.83} \mathrm{Ga}_{0.17} \mathrm{As}_{0.37} \mathrm{P}_{0.63}$ & 1.13 & 15 & $\mathrm{nid}$ \\
QW $(\times 6)$ & $\mathrm{In}_{0.78} \mathrm{Ga}_{0.22} \mathrm{As}_{0.79} \mathrm{P}_{0.21}$ & 1.55 & 8 & $\mathrm{nid}$ \\
SCH & $\mathrm{In}_{0.83} \mathrm{Ga}_{0.17} \mathrm{As}_{0.37} \mathrm{P}_{0.63}$ & 1.13 & 190 & $\mathrm{nid}$ \\
Interlayer & $\mathrm{InP}$ & 0.92 & 580 & $\mathrm{n}+$ \\
Etch-stop & $\mathrm{In}_{0.9765} \mathrm{Ga}_{0.0235} \mathrm{As}_{0.05} \mathrm{P}_{0.95}$ & 0.94 & 20 & $\mathrm{n}+$ \\
Interlayer & $\mathrm{InP}$ & 0.92 & 200 & $\mathrm{n}+$ \\
Bus core & $\mathrm{In}_{0.83} \mathrm{Ga}_{0.17} \mathrm{As}_{0.37} \mathrm{P}_{0.63}$ & 1.13 & 800 & $\mathrm{n}+$ \\
n-contact & $\mathrm{InP}$ & 0.92 & 2000 & $\mathrm{n}+$ \\
Substrate & $\mathrm{InP}$ & 0.92 & - & $\mathrm{n}$ \\
\hline \hline
\end{tabular}

rings beyond $30 \mu \mathrm{m}$ seems to vanish into a radiation mode. In the coupling region (2), the upper ring mode couples evanescently to the lower bus whispering gallery mode. The disk structure for the bus waveguide (3) avoids the need for the inner sidewall of the waveguide, simplifying drastically the placement of the ring over it. Finally, the bent bus waveguide is laterally tapered in order to guide the light into a single-mode rib structure (4). The entire design process is described in detail in [20].

The epitaxial layer structure is detailed in Table I. It essentially consists of a $0.8-\mu \mathrm{m}$-thick InGaAsP layer (with a bandgap cutoff wavelength of $\lambda_{\mathrm{g}}=1.13 \mu \mathrm{m}$ ), a $0.8-\mu \mathrm{m}$-thick InP separation layer, and a multiquantum-well (MQW) active core. The MQW region is composed of six $80-\AA$-thick $1 \%$ compressively strained InGaAsP $\left(\lambda_{\mathrm{g}}=1.55 \mu \mathrm{m}\right)$ quantum wells separated by 150 - $\AA$-thick $\operatorname{InGaAsP}\left(\lambda_{\mathrm{g}}=1.13 \mu \mathrm{m}\right)$ barriers and sandwiched between two 190-nm-thick InGaAsP $\left(\lambda_{\mathrm{g}}=1.13 \mu \mathrm{m}\right)$ separate confinement heterostructure ( $\mathrm{SCH}$ ) layers.

The epitaxial layers were grown by metal-organic chemical vapor deposition (MOCVD). The $\mathrm{SiO}_{\mathrm{x}}$ pattern of the rings was defined by plasma etching with conventional photoresist. The circular waveguides were etched by RIE through the active core down to the InP interlayer. Next, a selective wet etch down to the etch-stop layer using $\mathrm{H}_{3} \mathrm{PO}_{4}: \mathrm{HCl}$ (7:3) was performed to better control the depth and to reduce the lateral roughness of the ring ridge. Subsequently, the bus waveguide was dry etched using the above-mentioned chemistry and parameters. Thereafter, polyimide was spin-coated on the sample to form an insulation layer, and removed on the ridges by plasma etch. Next, using a negative photoresist and a metal liftoff process, the metallization pattern was defined. Finally, the substrate was thinned and a back contact was deposited.

A set of microring lasers with ring radii ranging from 5 to $80 \mu \mathrm{m}$ and a fixed bus disk radius of $100 \mu \mathrm{m}$ were fabricated. The lateral shift between the ring and the underlying bus disk waveguide was designed at $0.5 \mu \mathrm{m}$ for every device as a minimum defined by technology. The expected coupling factor is $4 \%$ for the smallest ring and $12 \%$ for the largest. Higher lateral misalignments imply an exponential decrease in the coupling coefficient, being reduced by half for a step of $1 \mu \mathrm{m}$ (see [20] for a more detailed quantitative analysis). SEM pictures of the cross 


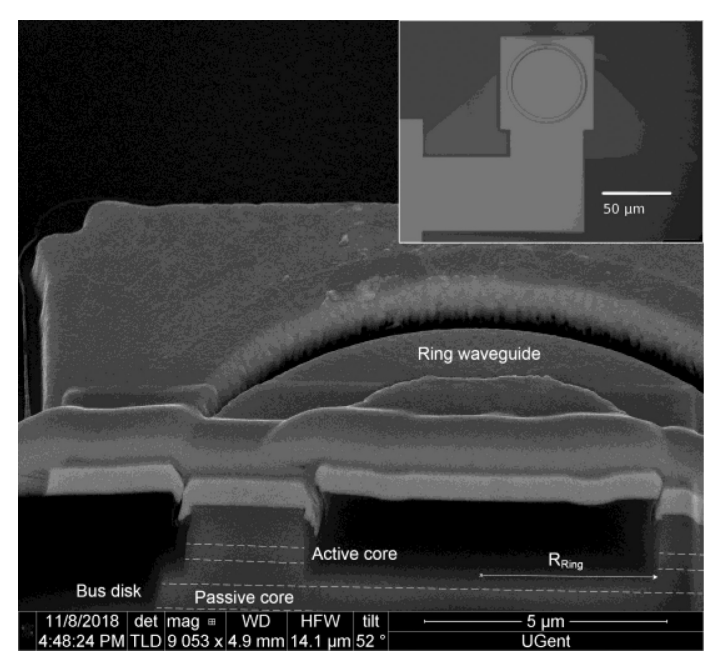

Fig. 2. SEM image of the cross section of a microring laser. The inset shows a top view photograph of the device after metallization.

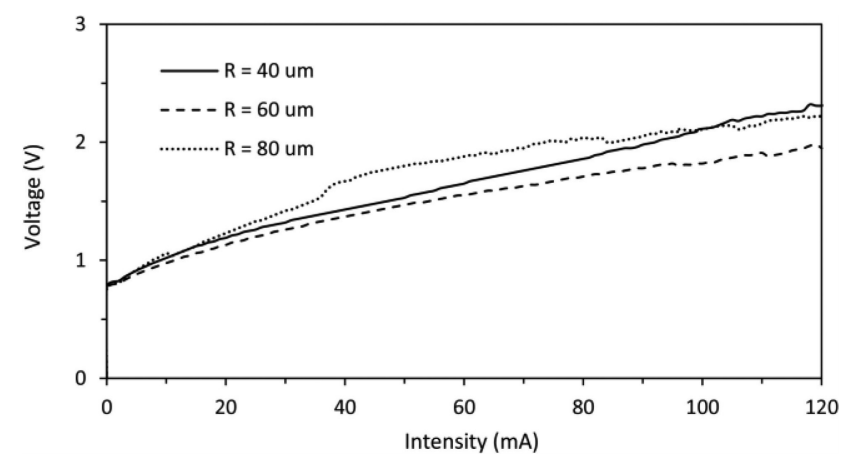

Fig. 3. V-I response of three microring diode lasers.

section of the device (Fig. 2) reveal that such submicron feature was fabricated reproducibly and measured $0.54 \mu \mathrm{m}$, implying a decrease of the coupling factor lower than $1 \%$ in the worst case. Pictures also confirm that the metal contact over the upper ring ridge was good.

\section{DEVICE RESULTS}

The devices were operated in CW regime at room temperature. Fig. 3 displays some typical electrical characteristics of the microring diodes. The devices show a low series resistance of around $20 \Omega$ at forward bias proving a good electrical performance. Fig. 4 shows the light intensity versus current density curves $(L-I)$ of the lasers, measured in one bus waveguide. The threshold current density decreases from 4.2 to $3.3 \mathrm{kA} / \mathrm{cm}^{2}$ for radii varying from 40 to $80 \mu \mathrm{m}$, as expected for higher coupling coefficients. The external efficiency also improves from 0.5 to $0.7 \mathrm{~mW} / \mathrm{A}$ for the same radius range. Some curves exhibit small ripples corresponding to longitudinal mode hopping in the amplifying ring section. The inset shows a shift of $6 \mathrm{~nm}$ in the optical spectrum for a ring of $80 \mu \mathrm{m}$ at both sides of one of these folds. Such multimode lasing spectra could be avoided defining a lateral grating on the ring waveguide during lithography in

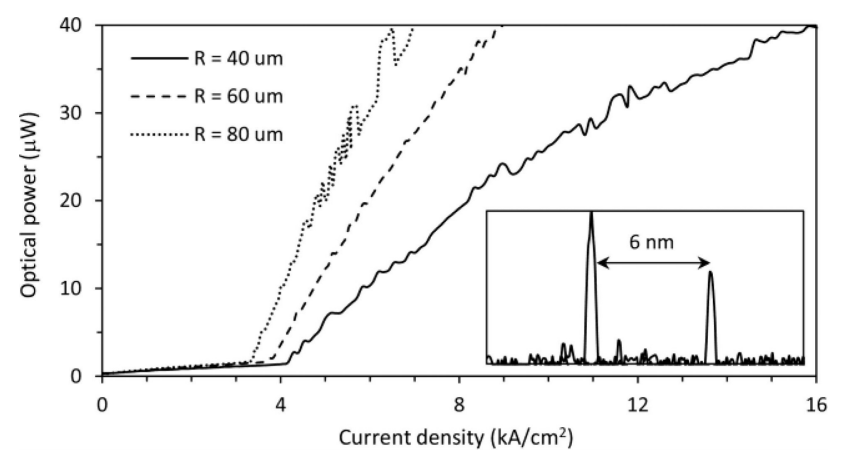

Fig. 4. Typical L-I characteristics of microring lasers operated in CW regime at room temperature and for three different ring radii.

order to favor the desired mode [21]. Lasing was observed for temperatures up to $60{ }^{\circ} \mathrm{C}$.

Nevertheless, in absolute terms, the lasers emitted less optical power than expected. Using pulsed current injection ( $2 \mu$ s pulse width and $2 \%$ duty cycle), the power increases to several tenths of milliwatts due to a better thermal performance in this regime, but these values are still lower than predicted. Furthermore, devices with the ring radius smaller than $40 \mu \mathrm{m}$ did not exhibit lasing. In addition to sidewall roughness in such highly confined structures, surface recombination of injected carriers is one of the major limiting factors to good laser performance. Therefore, optimizing the dry etch process and coating the etched surface with a layer of $\mathrm{SiO}_{2}$ or passivating it with $\mathrm{Al}_{2} \mathrm{O}_{3}$ by atomic layer deposition (ALD) [22] are expected to drastically reduce these sources of loss. The use of quantum dots (QDs) instead of quantum wells as the active media has also shown to be a good strategy to minimize the recombination on the resonator surface and achieve lower threshold lasing in small volume cavities [23]. Furthermore, since the analysis of structures with microbends is always difficult, even more so with two bend waveguides of different curvatures [20], the optical losses in the ring waveguide and the coupling region might be underestimated. Nevertheless, the technological simplicity of the structure allows overcoming the mentioned drawbacks without adding extra steps to the manufacturing procedure. Thus, in the ring section, by limiting the underlying slab passive waveguide to a broad (but finite) mesa waveguide, the asymmetry between the waveguides is preserved and the bend losses can be reduced, as proposed in [20]. On the other hand, the inefficiency of the coupler in small rings might be improved by choosing the same curvature for the underlying disk bus waveguide and the ring guide, thus increasing the coupling length and the efficiency. Future devices with smaller diameters and improved processing are expected to result in lower thresholds and higher power operation.

Fig. 5 depicts the measured output spectra of the working microring lasers, for different pump injection currents. The lasers show single-mode operation with a side-lobe suppression ratio higher than $30 \mathrm{~dB}$ for these operating conditions. Such performance might be due to dependency of the bending losses of the strongly asymmetric mode in the ring with the wavelength. Due to the total lateral symmetry of the structure, the lasers generate light in both opposite directions, exciting 

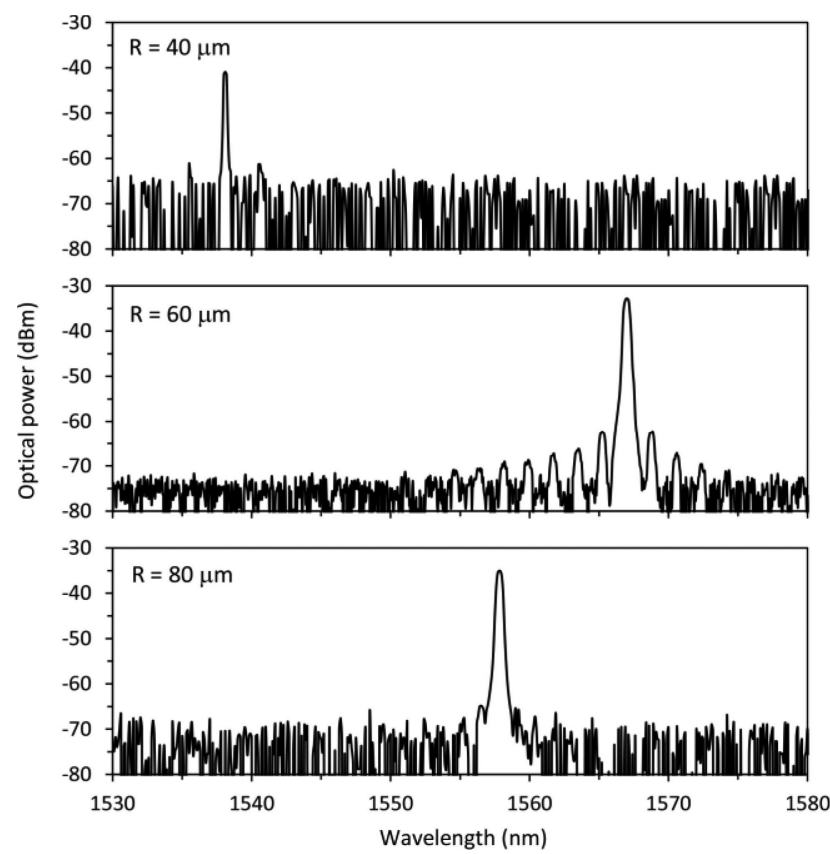

Fig. 5. The output spectra of the microring lasers for three different ring radii.

counter-propagating degenerate modes that couple to each other through scatterings or imperfections. If unidirectional lasing is required by the application, several technological strategies that are compatible with the structure of these lasers could be used, such as the definition of crossover S-bend waveguides in the ring cavity [24].

\section{CONCLUSION}

In this work, a new vertical microring laser using a single epitaxial growth on InP and conventional single-side lithography has been successfully demonstrated. The approach is based on two vertically coupled cores close to phase matching condition and avoids the need of complex fabrication processes such as electron beam lithography, wafer bonding or epitaxial regrowth. The lasers with radii bigger than $40 \mu \mathrm{m}$ show good electrical and optical characteristics. Smaller rings do not reach lasing due to surface recombination, propagation losses in the cavity and the reduced coupling factor because of the radius mismatch between the upper ring and the underlying disk waveguide. Such drawbacks can be easily overcome defining a broad rib waveguide in the bus core layer under the ring and reducing the curvature of the bus disk in the coupling region. Such improvements would not increase the complexity of the fabrication scheme.

\section{REFERENCES}

[1] L. Xu et al., "Silicon-on-insulator-based microwave photonic filter with widely adjustable bandwidth," Photon. Res., vol. 7, no. 2, pp. 110-115, Feb. 2019.

[2] B. Midya et al., "Supersymmetric microring laser arrays," Photon. Res., vol. 7, no. 3, pp. 363-367, Mar. 2019.

[3] H. Ren et al., "Dissipative sensing with low detection limit in a selfinterference microring resonator," J. Opt. Soc. Amer. B., vol. 36, no. 4, pp. 942-951, Apr. 2019.
[4] S. Pal and S. Gupta, "Performance analysis of an electrostatic doping assisted silicon microring modulator," Opt. Commun., vol. 430, pp. 131138, Jan. 2019.

[5] L. Liu et al., "Compact and broadband optical add-drop de-multiplexer with cascaded elliptical micro-rings on SOI," IEEE Photon. Tech. Lett., vol. 31, no. 6, pp. 451-454, Mar. 2019.

[6] Q. Cheng et al., "Scalable microring-based silicon Clos switch fabric with switch-and-select stages," IEEE J. Sel. Topics Quantum Electron., vol. 25, no. 5, Sep./Oct. 2019, Art. no. 3600111.

[7] M. Zhang et al., "Broadband electro-optic frequency comb generation in a lithium niobate microring resonator," Nature, vol. 568, pp. 373-377, Mar. 2019.

[8] A. Godbole et al., "All optical scalable logic gates using $\mathrm{Si}_{3} \mathrm{~N}_{4}$ microring resonators," IEEE J. Sel. Topics Quantum Electron., vol. 22, no. 6, Nov./Dec. 2016, Art. no. 5900308

[9] J. Zhang et al., "Optical dynamic memory based on an integrated active ring resonator," Opt. Lett., vol. 43, no. 19, pp. 4687-4690, Oct. 2018.

[10] D. Liang et al., "Electrically-pumped compact hybrid silicon microring lasers for optical interconnects," Opt. Express, vol. 17, no. 22, pp. 2035520364, Oct. 2009.

[11] C. W. Tee, K. A. Williams, R. V. Penty, and I. H. White, "Fabricationtolerant active-passive integration scheme for vertically coupled microring resonators," IEEE J. Sel. Topics Quantum Electron., vol. 12, no. 1, pp. 108116, Jan./Feb. 2006.

[12] M. Smit et al., "An introduction to InP-based generic integration technology," Semicond. Sci. Technol., vol. 29, no. 8, 2014, Art. no. 083001.

[13] M. T. Hill et al., "A fast low-power optical memory based on coupled micro-ring lasers," Nature, vol. 432, pp. 206-209, Nov. 2004.

[14] V. M. Menon, F. Xia, and S. R. Forrest, "Photonic integration using asymmetric twin-waveguide (ATG) technology: Part II-Device," IEEE J. Sel. Topics Quantum Electron., vol. 11, no. 1, pp. 30-42, Jan./Feb. 2005.

[15] V. Tolstikhin, "Regrowth-free multi-guide vertical integration in InP for optical communications," in Proc. 23rd Int. Conf. Indium Phosphide Related Mater, Berlin, Germany, May 2011, pp. 281-284.

[16] V. M. Menon, W. Tong, and S. R. Forrest, "Control of quality factor and critical coupling in microring resonators through integration of a semiconductor optical amplifier," IEEE Photon. Technol. Lett., vol. 16, no. 5, pp. 1343-1345, May 2004.

[17] J. G. M. van der Tol et al., "Indium phosphide integrated photonics in membranes," IEEE J. Sel. Topics Quantum Electron., vol. 24, no. 1, Jan./Feb. 2018, Art. no. 6100809 .

[18] Y. Jiao et al., "First demonstration of an electrically pumped laser in the InP membrane on silicon platform," in Advanced Photonics 2015, OSA Technical Digest (online) (Optical Society of America, 2015), paper IM4B.3.

[19] O. G. López, D. Lasaosa, M. López-Amo, and M. Galarza, "Highly efficient fully resonant vertical couplers for InP active passive monolithic integration using vertically phase matched waveguides," Opt. Express, vol. 21, no. 19, pp. 22717-22727, Sep. 2013.

[20] O. G. López, D. Van Thourhout, D. Lasaosa, M. López-Amo, R. Baets, and M. Galarza, "Vertically coupled microring resonators using one epitaxial growth step and single-side lithography," Opt. Express, vol. 23, no. 4, pp. 5317-5326, Feb. 2015.

[21] A. Arbabi, S. M. Kamali, E. Arbabi, B. G. Griffin, and L. L. Goddard, "Grating integrated single mode microring laser," Opt. Express, vol. 23, no. 4, pp. 5335-5347, Feb. 2015.

[22] Y. Wan et al., "Low-threshold continuous-wave operation of electrically pumped $1.55 \mu \mathrm{m}$ InAs quantum dash microring lasers," ACS Photon., vol. 6, no. 2, pp. 279-285, Dec. 2018.

[23] Y. Wang et al., "Sub-wavelength InAs quantum dot microdisk lasers epitaxially grown on exact $\mathrm{Si}$ (001) substrates," Appl. Phys. Lett., vol. 108, no. 22, 2016, Art. no. 221101.

[24] J. Ren et al., "Unidirectional light emission in PT-symmetric microring lasers," Opt. Express, vol. 26, no. 21, pp. 27153-27160, Oct. 2018.

Óscar García López received the master's degree in Communications Technology and the Telecommunication Engineering degree from the Universidad Pública de Navarra in 2009 and 2008, respectively. He is a Telecom Engineer. He has authored papers concerning several topics such as fiber optic amplifiers, laser integration for optical communications, nanostructured surfaces for solar cell efficiency enhancement or biosensors based on Local Surface Plasmon Resonance (LSPR) phenomenon. At present, he is working on design, modeling and simulation for fluid dynamics systems and energy harvesting devices in NAITEC, a technology research center oriented to intelligence mobility and advanced manufacturing industry. 
Dries Van Thourhout received the degree in physical engineering and the Ph.D. degree from Ghent University, Ghent, Belgium, in 1995 and 2000, respectively. From October 2000 to September 2002, he was with Lucent Technologies, Bell Laboratories, New Jersey, USA, working on the design, processing, and characterization of InP/InGaAsP monolithically integrated devices. In October, 2002 he joined the Department of Information Technology, Ghent University, Belgium. He is a member of the Permanent Staff of the Photonics group. Since 2008, he has been a Full Time Professor. He is a lecturer for four courses within the Ghent University (Microphotonics, Advanced Photonics Laboratory, Photonic Semiconductor Components, and Technology). He is coordinating the cleanroom activities of the research group and is the coordinator of the NAMIFAB centre of expertise. He has submitted 14 patents, has authored and coauthored more than 220 journal papers, and has presented invited papers at all major conferences in the domain. His research focuses on the design, fabrication, and characterization of integrated photonic devices. Main topics involve Silicon nanophotonic devices and the integration of novel materials (III-V, graphene, ferro-electrics, quantum dots,...) on these waveguides to expand their functionality. He is working on applications for telecom, diatom, optical interconnect, and sensing. He is a member of IEEE Photonics Society, OSA, and SPIE. He has coordinated several European Projects (FP6 PICMOS, FP7 WADIMOS, and FP7 SMARTFIBER), contributed to many more, and received an ERC Grant (ULPPIC). He received the prestigious "Laureaat van de Vlaamse Academie Van Belgie" prize in 2012 and was a Clarivate highly cited researcher.

Steven Verstuyft is responsible for the processing of optoelectronic devices with the Department of Information Technology of the University of Ghent, Gent, Belgium.

Manuel López-Amo (Senior Member, IEEE) has been a Full Professor in photonics with Universidad Pública de Navarra, Pamplona, Spain,since 1996. $\mathrm{He}$ is the the Head of the Optical Communications group there. He is the author of more than $300 \mathrm{JCR}$ peer reviewed technical articles and international conferences. He has led 31 research projects being the co-investigator in another 26 . His research interests are in optical fiber sensors, optical networks, optical fiber lasers, semiconductor lasers, and integrated optics. He is the Spanish representative at the experts committee IEC SC86C WG2 on Fiber Optic Sensors, and the European Cost Actions TD1001 "OFSeSa" and 299 "Fides." He has been an Associate Editor for the IEEE/OSA JOURNAL OF LIGHTWAVE TECHNOLOGY and at MDPI Sensors among others. He is a member of the OSA.
Roel Baets (Fellow, IEEE) received the first M.Sc. degree in electrical engineering from Ghent University (UGent), Ghent, Belgium, in 1980, the second M.Sc. degree from Stanford University, Stanford, CA, USA, in 1981, and the Ph.D. degree from UGent in 1984. From 1984 to 1989, he held a Post-Doctoral position with IMEC. Since 1989, he has been a Professor with the Faculty of Engineering and Architecture, UGent, where he founded the Photonics Research Group. From 1990 to 1994, he was a part-time Professor with the Delft University of Technology and from 2004 to 2008 with the Eindhoven University of Technology. He is currently a Full Professor with UGent and is associated with IMEC. He has mainly worked in the field of integrated photonics. He has made contributions to research on photonic integrated circuits, both in III-V semiconductors and in silicon, as well as their applications in telecom, datacom, sensing, and medicine. He is an ERC Grantee of the European Research Council and a Methusalem Grantee of the Flemish Government. He is a fellow of the European Optical Society and the Optical Society. He is also a member of the Royal Flemish Academy of Belgium for Science and the Arts.

Marko Galarza received the Telecommunications Engineering degree from the Universidad Pública de Navarra, Pamplona, Spain, in 1997 and the Ph.D. degree from the same university and the University of Ghent, Ghent, Belgium, in 2003. In 2004, he was a visiting Postdoctoral Researcher with the Photonics Research Group, Ghent University-IMEC, Ghent, Belgium, supported by a Marie Curie fellowship. In 2005, he joined the Optical Communications Group, Department of Electrical, Electronic and Communication Engineering, Universidad Pública de Navarra as an Assistant Professor. His research interests are fiber-optic sensors and integrated optics components, such as semiconductor waveguides, laser diodes and microring resonators. 